\title{
Réparation du tunnel de Vernier par voûte injectée
}

\section{Grouted soil arch for the reconstruction of the Vernier tunnel}

\author{
H. DUPLAINE et J.-L. RICHARD \\ SIF-BACHY*
}

Rev. Franç. Géotech. n 63, pp. $69-78$ (avril 1993)

\section{Résumé}

Le tunnel de Vernier, qui fera partie du contournement autoroutier de Genève s'est effondré pendant la construction des piédroits alors que la demi-section supérieure était terminée.

Le fontis ainsi créé est immédiatement remonté à la surface 32 mètres plus haut, créant un cratère de $1500 \mathrm{~m}^{3}$. La consolidation par injection de la zone à réexcaver augmentée d'une voûte de 8 mètres d'épaisseur, solution variante présentée par l'entreprise, a été choisie comme solution de réparation.

Les injections se sont déroulées en plusieurs phases, remplissage des vides, recompactage et imprégnation avec une gamme de coulis allant du simple mortier au coulis écologique MICRON S, le tout piloté par le système EPICEA qui a géré 14 points d'injection simultanés.

\section{Abstract}

The Vernier tunnel, one of the future Geneva by-pass highway structures collapsed during construction of the footwalls, the upper half section being already concreted.

A sinkhole immediately developed up to the surface, 32 meters higher, leaving a crater of $1500 \mathrm{~m}^{3}$. The recompaction through grouting of a vault 8 meters thicker than the section to reexcavate, an alternative solution proposed by the specialist contractor, was elected for the repair of the structure.

Several phases were needed to achieve the treatment : void filling, recompaction and impregnation using a wide range of grouts going from a simple mortar to environmentally safe mineral grout MICRON S. All the grouting was carried out using the EPICEA who has been able to drive 14 grouting points simultaneously. 


\section{INTRODUCTION}

Le tunnel de Vernier en Suisse, fait partie du projet de contournement autoroutier de Genève. L'ouvrage se compose de deux tubes de 1900 mètres de long et 12 mètres de large et séparés de 35 mètres d'axe en axe. L'accident s'est produit dans le tube Rhône à midistance des deux extrémités alors que la couverture, à cet endroit, était de 32 mètres.

La géologie du site est très représentative de la plaine genevoise à savoir (fig. 1):

- une couche de moraine würmienne, phase limoneuse sous les sols de couverture ou remblais;

- la couche des cailloutis morainiques profonds plus communément dénommée "alluvion ancienne » où l'on retrouve aussi bien des boulders que des inclusions de sable très fin;

- la molasse grise à gypse qui est essentiellement composée de marne. Cette roche tendre s'altère très vite après avoir été excavée et présente un caractère légèrement expansif ;

- la molasse rouge.

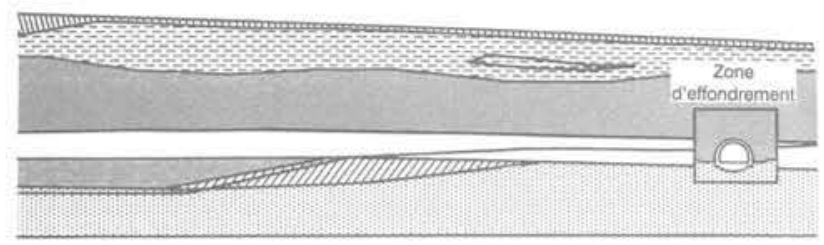

\begin{tabular}{|c|c|}
\hline MIIIIIIIII Rembiais, sols de couverture & DIIIZ Molasse grise a Gypse \\
\hline 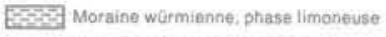 & W Molasse rouge \\
\hline
\end{tabular}

Fig. 1. - Géologie du site.

Fig. 1, - Site geology

Les molasses grises et rouges peuvent être localement très fracturées et traversées par des failles, ce qui augmente la sensibilité des marnes.

Il n'y a pas de nappe à proprement parler mais des circulations d'eau existent toutefois au niveau du contact entre les moraines et la molasse.

Les méthodes de construction employées furent choisies en fonction des conditions géologiques par des ingénieurs ayant une bonne pratique de la construction de tunnels dans la région. Les deux tubes furent creusés selon la méthode belge comme décrit dans la figure 2 . Du côté où l'intégralité de la demi-section supérieure se trouve dans la molasse, un trou pilote fut d'abord excavé à l'aide d'un tunnelier d'un diamètre de 3,5 mètres et fut ensuite porté aux dimensions définitives à l'aide d'une machine d'attaque ponctuelle. Du côté opposé où la demi-section supérieure se trouve entièrement dans la moraine, l'excavation et le bétonnage ont été réalisés à l'abri d'un bouclier à lances, permettant un travail aux dimensions finales. Dans les deux cas le stross était ensuite excavé, puis les piédroits construits par longueurs de 7,5 mètres avec report des charges induites par la voûte de part et d'autre de la

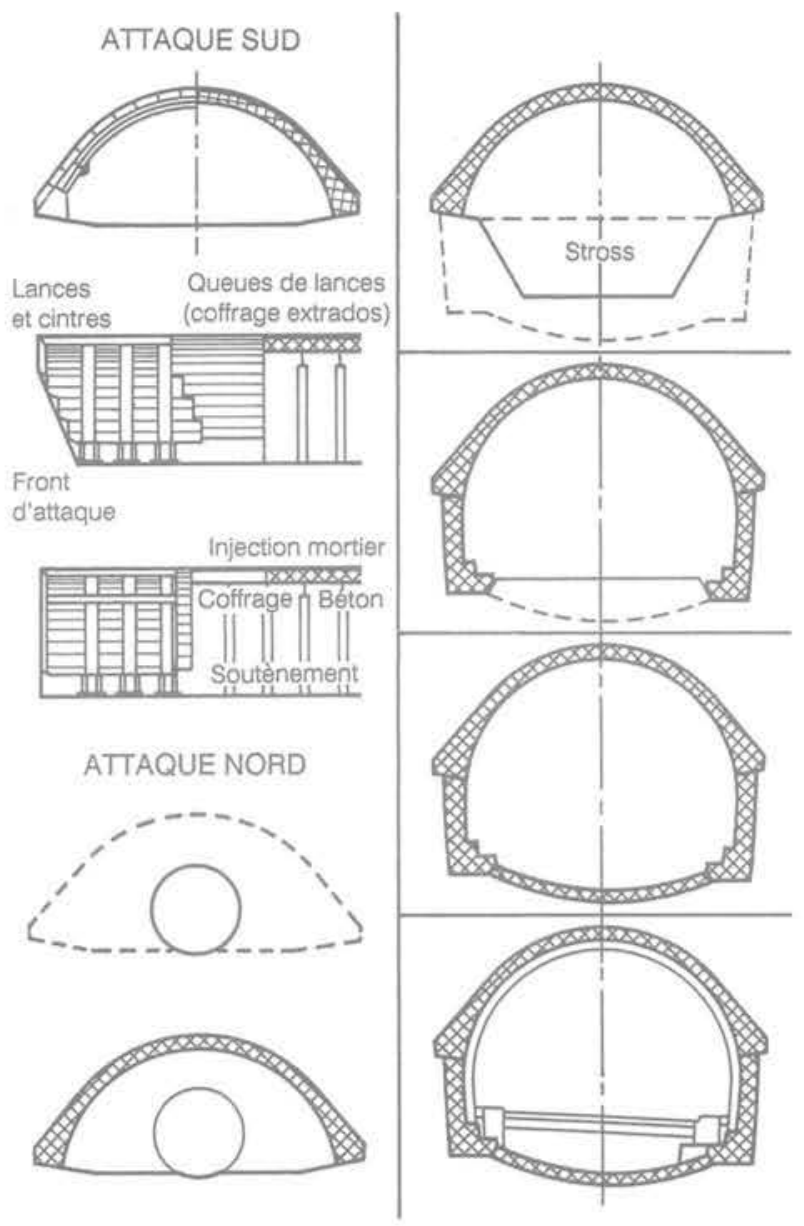

Fig. 2. - Méthodes de construction du tunnel.

Fig. 2. - Tunnel construction methods.

zone à construire au moyen d'une poutre continue en béton armé. Enfin, un radier complétait l'ouvrage dans les zones où la route reposait directement sur la molasse grise afin de prendre en compte son caractère expansif.

\section{DESCRIPTION DE L'ACCIDENT}

Le 10 avril 1990 lors de la construction des 100 derniers mètres de piédroits du tube Rhône, alors que le tube Jura était terminé, une rupture de la molasse a entraîné un effondrement de la voûte en béton. L'accident a eu lieu dans la section centrale du tunnel où la voûte était fondée sur l'interface molasse/moraine. L'effondrement a eu lieu deux heures après les premiers signes de désordre laissant tout juste le temps au personnel d'évacuer la zone.

Le fontis amorcé par l'effondrement de la voûte est immédiatement remonté à la surface, 32 mètres plus haut. En surface le cratère avait un diamètre de 25 mètres et une profondeur de 5 à 7 mètres. La voûte s'était effondrée sur 55 mètres et le tunnel partiellement remblayé par $3500 \mathrm{~m}^{3}$ de matériaux décomprimés. La figure 3 résume sous forme de schéma la géométrie de l'accident: y figurent également les mesures conforta- 


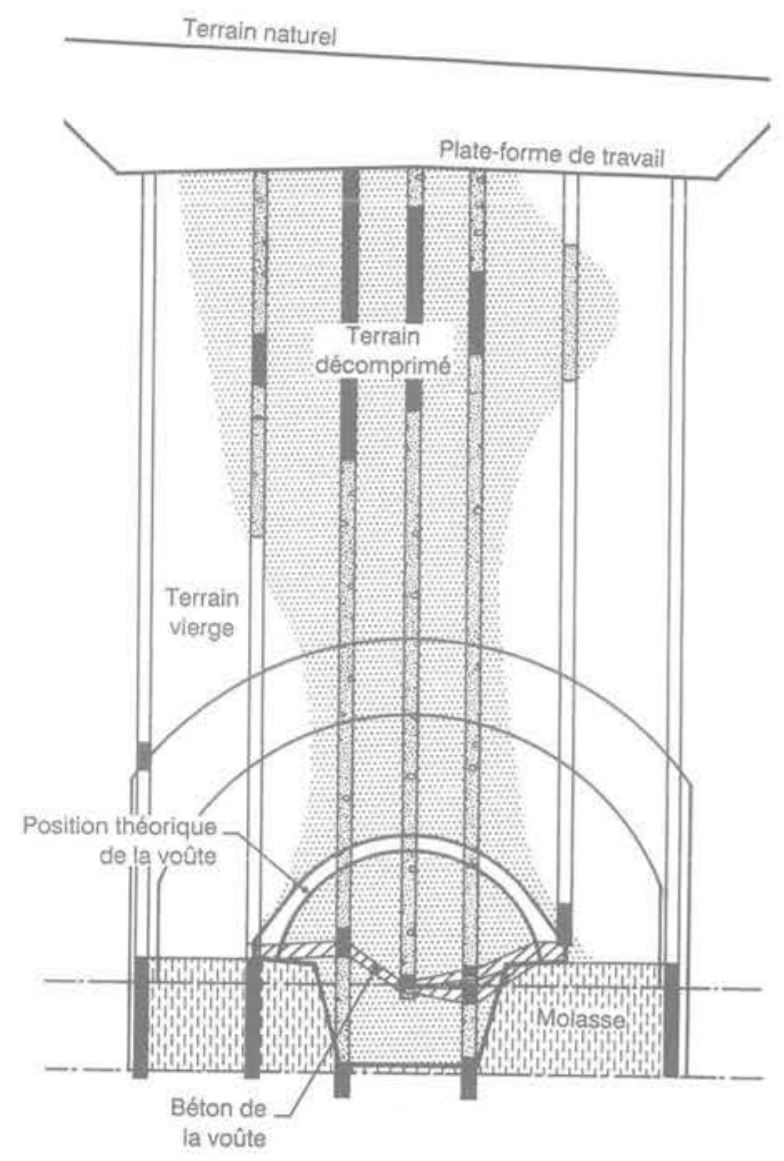

Fig. 3. - Géométrie de l'accident.

Fig. 3. - Geometrical characteristics of the collapse.

tives immédiates telles que risbermes de stabilité et murs-masque bloquant la propagation de l'effondrement.

Plusieurs solutions techniques permettant la reprise du percement furent évaluées par la maîtrise d'œuvre, et c'est une solution injection depuis la surface qui fut choisie par le maitre d'ouvrage comme base d'appel d'offres. Un consortium mené par SIF-GROUTBOR, filiale de SIF-BACHY, proposa une variante basée sur une voûte injectée de 6 mètres d'épaisseur, dont 3 mètres résistants, grâce à son expérience et à sa capacité à assurer la qualité des travaux par un pilotage des injections par ordinateur au moyen du système EPI. CEA. Sur les conseils du professeur F. DESCCEUDRES, le maître d'ouvrage demanda que l'épaisseur de la voûte résistante soit portée à 5 mètres, pour une voûte injectée de 8 mètres d'épaisseur totale. Un contrat fut finalement passé au consortium fin août 1990, pour une mobilisation le 15 septembre 1990, soit environ 6 mois après l'accident. La durée des travaux était fixée à 6 mois.

\section{PRINCIPE DU TRAITEMENT}

Le pilote technique du consortium, exécutant les injections, a proposé un traitement que l'on trouve résumé en figure 4 et basé sur le phasage suivant:

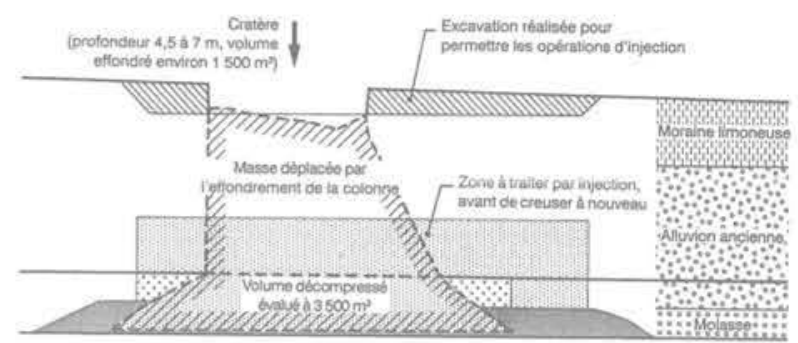

Fig. 4. - Phasage du traitement.

Fig. 4. - Treatment phases.

\subsection{Phase 1}

Réalisation de 20 forages de reconnaissance répartis sur 2 à 3 lignes. Deux paramètres de forage furent systématiquement enregistrés: la poussée sur l'outil (maintenue constante) et la vitesse d'avancement instantanée de l'outil de forage, afin de dresser une cartographie des vides et des zones décomprimées. Un exemple de coupe longitudinale obtenue à partir de ces enregistrements est donné en figure 5. Ces forages étaient descendus un mètre au-dessous du niveau précédemment excavé et équipés d'un tube lanterné permettant d'injecter les vides mis en évidence par l'interprétation des enregistrements de paramètres de forage.

\subsection{Phase 2}

Remplissage des vides au moyen d'un mortier dont la composition était :

Ciment CLK $45: 250 \mathrm{~kg} / \mathrm{m}^{3}$

Bentonite: $24 \mathrm{~kg} / \mathrm{m}^{3}$

Filler: $800 \mathrm{~kg} / \mathrm{m}^{3}$

Eau : $629 \mathrm{l} / \mathrm{m}^{3}$

Au total, $490 \mathrm{~m}^{3}$ de ce mortier ont été injectés au travers des tubes lanternés des 20 forages de reconnaissance, en respectant les critères d'injection suivants:

- pas de limite sur le volume,

- pression de $3 \mathrm{MPa}$ en sortie de presse.

$\mathrm{Au}$ cours de cette phase, on a pu observer des résurgences en galerie au niveau des deux fronts. Le niveau de résurgence est progressivement monté, en cours de traitement, des naissances de voûte aux clés de voûte, ce qui est une indication sur le bon remplissage des vides.

\subsection{Phase 3}

Réalisation des forages primaires sur une maille de $2,5 \mathrm{~m}$ par $2,6 \mathrm{~m}$ descendus jusqu'à la cote précédemment excavée. Des tubes à manchettes en PVC renforcé d'un diamètre intérieur de 1 ' $1 / 2$ et équipés de 3 manchettes au mètre étaient mis en place dans les forages à l'abri d'un tubage provisoire. Ils furent scellés au terrain à l'aide d'un coulis de gaine dosé à $250 \mathrm{~kg} / \mathrm{m}^{3}$ de ciment. 

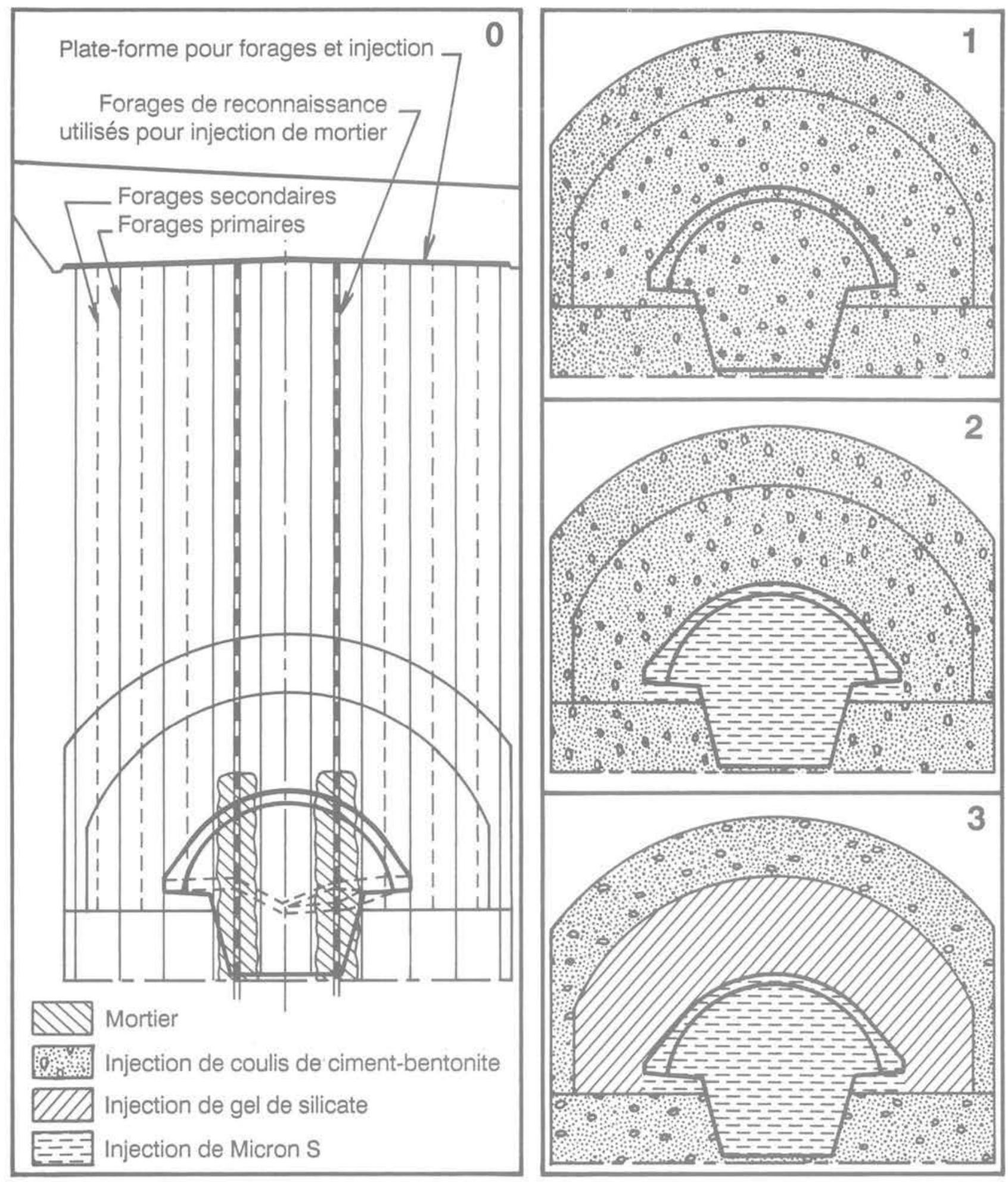

Fig. 5. - Exemple de cartographie basée sur les diagraphies.

Fig. 5. - Example of mapping from diagraphies.

\subsection{Phase 4}

Première phase de recompaction dans les forages primaires, sur toute la section du traitement, soit jusqu'à 8 mètres au-dessus de la section à réexcaver, à l'aide d'un coulis bentonite-ciment dont la composition suit :
Ciment CLK $45: 350 \mathrm{~kg} / \mathrm{m}^{3}$

Bentonite : $33,5 \mathrm{~kg} / \mathrm{m}^{3}$

Eau : $867 \mathrm{l} / \mathrm{m}^{3}$

Dans les zones recevant un autre type d'injection ulté. rieurement, seule une manchette sur deux était injectée 
alors que toutes les manchettes étaient injectées ailleurs. Les critères d'injection étaient:

- volume limité à $900 \mathrm{l} / \mathrm{ml}$ (1 050 pour les lignes extérieures) ;

- pression limitée à $1,5 \mathrm{MPa}$ pour les zones recevant un autre type d'injection et $2 \mathrm{MPa}$ ailleurs;

- débit minimum de $350 \mathrm{l} / \mathrm{h}$ à la pression maximum; - réinjection immédiate, si le volume maximum est atteint, avec une pression finale inférieure à la moitié de la pression maximum.

D'importantes résurgences ont été constatées en galerie sur les deux fronts, ce qui est tout à fait normal dans ce type de traitement, bien que les forages périphé. riques aient été traités en premier.

\subsection{Phase 5}

Réalisation des forages secondaires situés sur les centres du maillage primaire ramenant ainsi la maille finale des forages à environ $1,8 \mathrm{~m}$ par $1,8 \mathrm{~m}$. Contrairement aux primaires, les forages secondaires sont arrêtés à l'interface molasse/moraine, la maille primaire ayant été estimée suffisante pour le traitement de la molasse.

En raison des mouvements importants qui pouvaient être prévus pendant l'injection des forages primaires, il a été décidé d'attendre que ceux-ci soient injectés avant d'entreprendre le forage des secondaires, sous peine de ne pouvoir y descendre les obturateurs doubles le moment venu.

\subsection{Phase 6}

Seconde phase de recompactage, en utilisant le même coulis bentonite-ciment que précédemment, la même répartition de manchettes traitées, et en traitant toute la section, jusqu'à 8 mètres au-dessus de la zone réexcavée, avec les critères d'injection suivants:

- volume limité à $450 \mathrm{l} / \mathrm{ml}$ (150 pour la périphérie) ; - pression maximum de $2 \mathrm{MPa}$ dans les zones ne recevant pas d'autre traitement et $3 \mathrm{MPa}$ ailleurs :

- débit minimum de $350 \mathrm{l} / \mathrm{h}$ à la pression maximum; - réinjection dans la foulée, si le volume limite est atteint, avec une pression finale inférieure à la moitié de la pression maximum.

\subsection{Phase 7}

Une fois la phase de recompactage terminée, le traitement est completé par une injection d'imprégnation de la zone à réexcaver et d'une voûte de $5 \mathrm{~m}$ l'entourant. Cette injection avait pour but de donner de la cohésion aux inclusions de sable fin pouvant être présentes dans la voûte résistante.

Deux types de coulis, possédant des caractéristiques de pénétrabilité équivalentes furent utilisés. L'un est le MICRON S, coulis minéral, l'autre un gel de silicate avec réactif organique.

Le coulis minéral fut injecté en premier dans la section devant être réexcavée afin que les matériaux n'aient pas à être stockés en décharge contrôlée du fait de la pré. sence de réactif organique. Le gel de silicate fut injecté dans la voûte porteuse, la nappe phréatique étant pauvre et inexploitée.

Ce coulis minéral est composé de fumée de silice et de chaux micronisée. Il a été développé pour répondre aux interdictions de plus en plus fréquentes en Europe frappant les gels de silicate utilisant comme réactif des durcisseurs d'origine organique qui sont les seuls à permettre d'obtenir des gels de consolidation.

Bien que n'étant pas un liquide pur comme le gel de silicate, il comporte $75 \%$ de particules inférieures à $1 \mu \mathrm{m}$ (fig. 6), les plus grossières $10 \mu \mathrm{m}$, ce qui lui confere pratiquement le même pouvoir de pénétration qu'un gel de silicate de formulation courante et, dans tous les cas, qu'un coulis à base de ciment super-fin. La figure 7 rappelle les domaines d'utilisation des différents grands groupes de coulis.

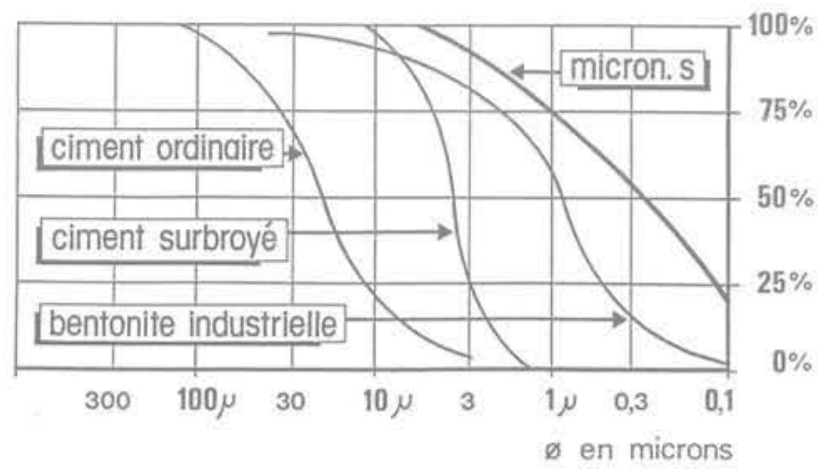

Fig. 6. - Granulométrie du coulis MICRON S.

Fig. 6. - Grain size distribution of MICRON S grout.

A poids de matière sèche égal, comme le montre la figure 8, le MICRON S présente des résistances méca. niques supérieures à celles de coulis à base de ciment CLK ou CPA et a fortiori de celles des gels de silicate. Sur ce chantier le dosage en matière sèche était de $150 \mathrm{~kg} / \mathrm{m}^{3}$ donnant ainsi des résistances à la compression simple sur coulis pur de $1 \mathrm{MPa}$ à 28 jours, soit plus de $2 \mathrm{MPa}$ dans le terrain traité.

Les critères d'injection du MICRONS furent les suivants :

- volume limité à $600 \mathrm{l} / \mathrm{ml}$,

- pression maximum de $3 \mathrm{MPa}$,

- débit minimum de $300 \mathrm{l} / \mathrm{h}$ à la pression maximum.

La composition du gel de silicate a été déterminée en prenant en considération les hypothèses suivantes:

- pérennité de 6 mois ;

- résistance sur éprouvette AFTES de $1 \mathrm{MPa}$ (pour viser 1,5 à $2 \mathrm{MPa}$ en terrain traité);

ce qui a conduit au choix suivant:

- volume d'eau sur volume de silicate $=1$,

- poids de réactif sur volume de silicate $=0,12$, soit :

- silicate de soude: $475 \mathrm{l} / \mathrm{m}^{3}$;

- eau : $475 \mathrm{l} / \mathrm{m}^{3}$;

- réactif $600 \mathrm{~B}: 50 \mathrm{l} / \mathrm{m}^{3}$. 


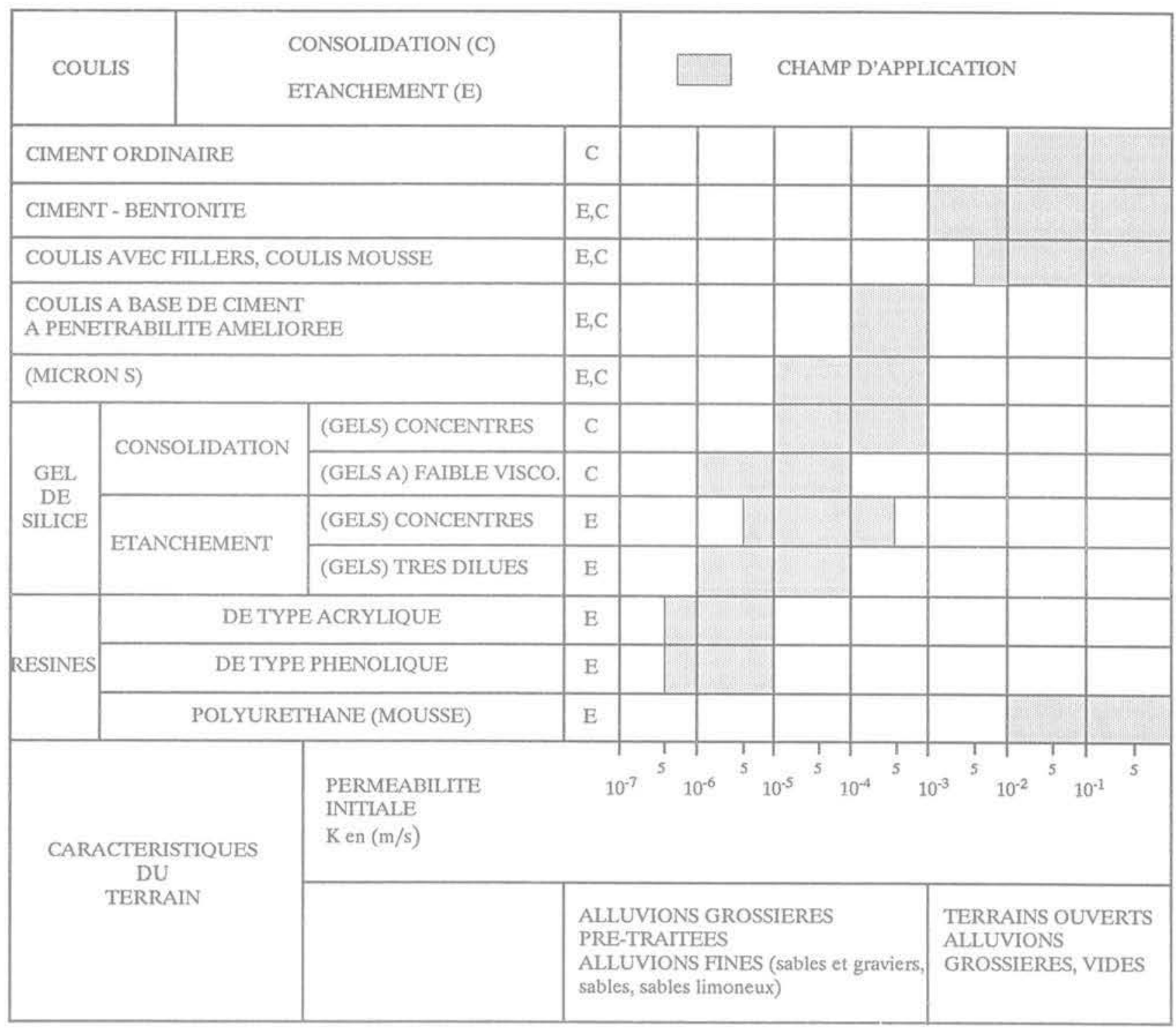

Fig. 7. - Domaines d'utilisation des différents coulis.

Fig. 7 - Grout selection table.

L'injection du gel de silicate dans toutes les manchettes de la voûte fut menée en respectant les critères d'injection suivants :

- volume limité à $1200 \mathrm{l} / \mathrm{ml}$ sur les primaires, $600 \mathrm{l} / \mathrm{ml}$ sur les secondaires;

- pression maximum $2 \mathrm{MPa}$;

- débit minimum de $300 \mathrm{l} / \mathrm{h}$ à pression maximum.

Des forages tertiaires furent injectés après détection de zones de faible absorption.

\section{TRAITEMENT}

Les quantités totales d'injection furent les suivantes:

- mortier : $490 \mathrm{~m}^{3}$;

- bentonite-ciment primaires : $2080 \mathrm{~m}^{3}$, secondaires : $834 \mathrm{~m}^{3}$;
- MICRON S: $404 \mathrm{~m}^{3}$;

- gel de silicate $\mathrm{P}+\mathrm{S}: 455 \mathrm{~m}^{3}$; tertiaires : $571 \mathrm{~m}^{3}$.

Au total $5500 \mathrm{~m}^{3}$ de coulis ont été injectês, $18250 \mathrm{ml}$ de forages réalisés pour reconsolider $18600 \mathrm{~m}^{3}$ de terrain, ce qui représente une incorporation moyenne de $29,5 \%$ du volume traité soit légèrement moins que la porosité de la moraine intacte qui est de 30 à $35 \%$. Les figures 9 et 10 donnent une idée plus précise de la distribution géographique des incorporations.

\section{SUIVI DU TRAITEMENT}

Afin de s'assurer de la réussite du traitement permettant ainsi de garantir la sécurité des ouvriers pendant la phase de recreusement, il était indispensable de mettre en ceuvre une possibilité de suivre très précisément l'évolution de celui-ci. 


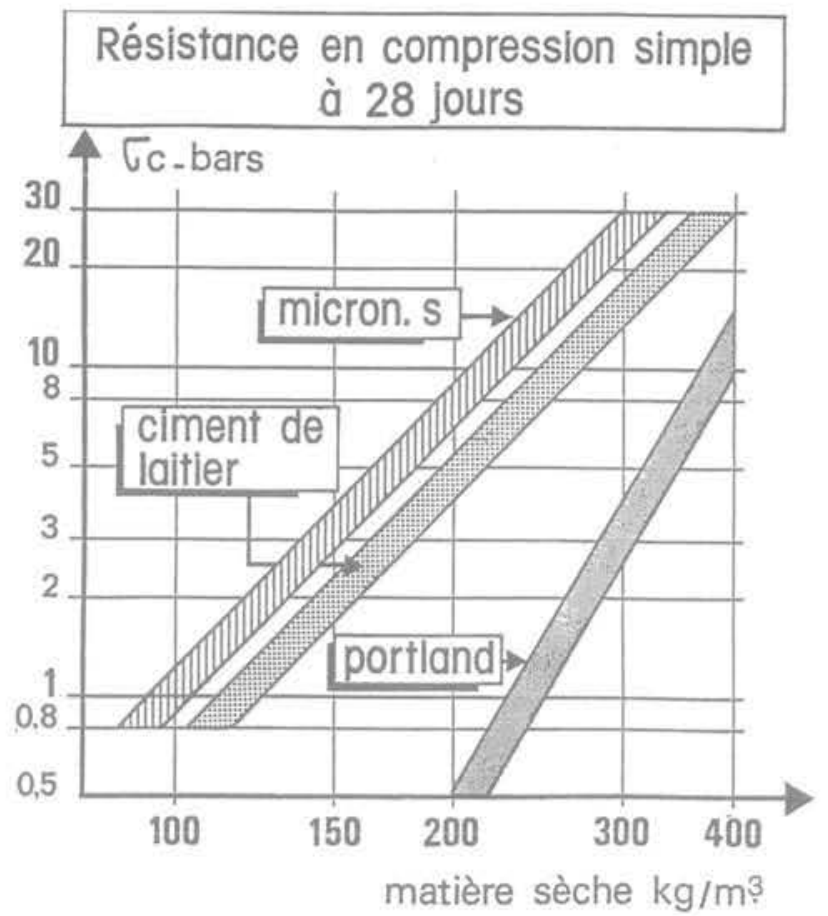

Fig. 8. - Résistance à la compression simple du coulis MICRON S.

Fig. 8. - Unconfined compression strength of MICRON S grout.

Pourcentages de traitement dans la zone bentonite-ciment

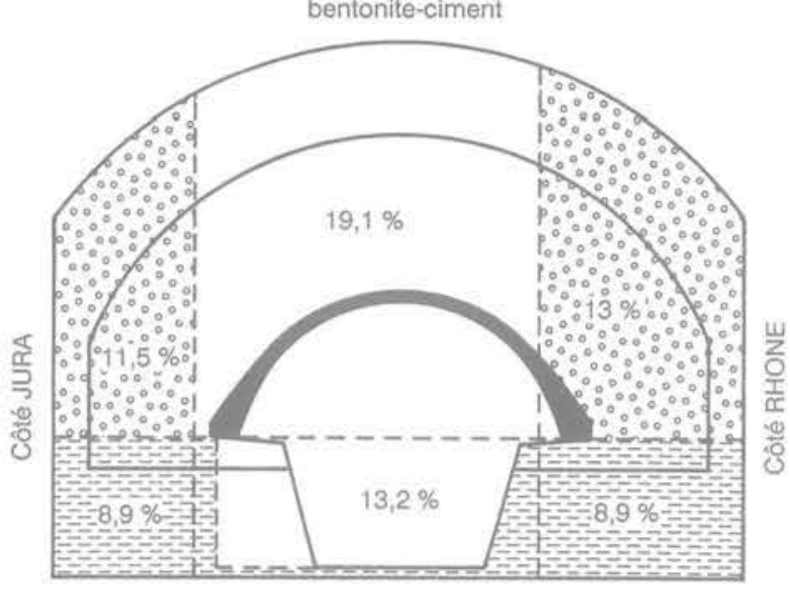

$\because \because$ Terrain de couverture en place

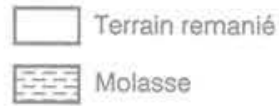

Fig. 9. - Distribution des absorptions en bentonite/ciment.

Fig. 9. - Grout takes bentonite/ciment.

Une des mesures prises, et certainement la plus importante, fut d'utiliser le système EPICEA (Enregistrement des Paramètres d'Injection et Contrôle Electronique Asservi).

Ce système fut mis en ceuvre pour la première fois en 1987 et correspondait à une demande des maître

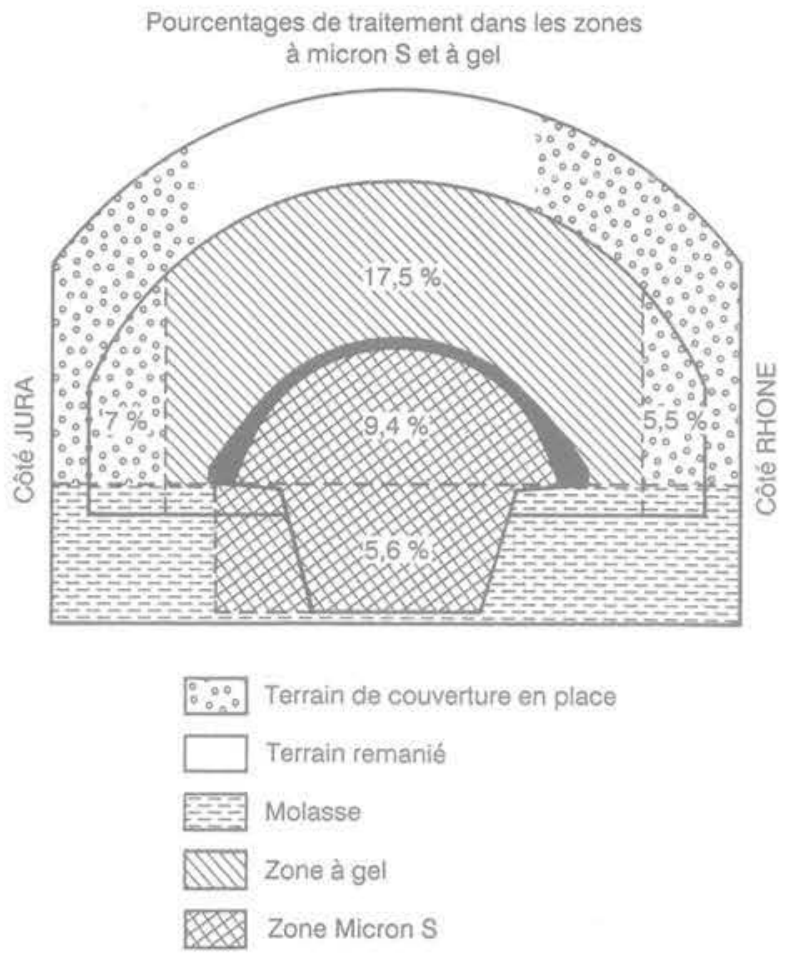

Fig. 10. - Distribution des absorptions en MICRON S/gel.

Fig. 10. - Grout takes MICRON S/silica gel

d'œuvre et maître d'ouvrage portant plus particulièrement sur l'enregistrement des volumes et pressions d'injection. Le système fut conçu pour aller au-delà de cette demande avec les trois fonctions principales suivantes:

- acquisition de données,

- asservissement des presses d'injection,

- construction d'une base de données permettant l'analyse immédiate des résultats.

Le volet acquisition est repris dans la figure 11 où l'on retrouve un micro-ordinateur alimenté par des débimètres électro-magnétiques et des capteurs de pression. Le système est prévu pour acquérir les données en provenance de 16 points d'injection. Le logiciel d'acquisition de données travaille en temps réel, ce qui permet d'avoir un échantillonnage d'une valeur par paramètre (pression, volume et débit) et par point d'injection par milliseconde. Les systèmes d'acquisition sous MS-DOS ne permettent pas un échantillonnage représentatif par manque de valeurs,

L'ordinateur arrête de lui-même les presses d'injection en cas de dépassement d'un critère d'injection préétabli, tel que pression ou volume maximum. Il peut également travailler en mode asservissement en respectant un débit de consigne ou gérer un refus, c'est-à-dire se maintenir en dessous d'une pression maximum en agissant sur le débit jusqu'à atteindre un débit minimum préétabli et maintenir alors l'injection à pression maximum et débit minimum pendant un temps t fixé à l'avance. Cette dernière possibilité est particulièrement intéressante dans les injections en rocher. Une des qualités du système est donc de s'affranchir des risques d'erreur humaine 


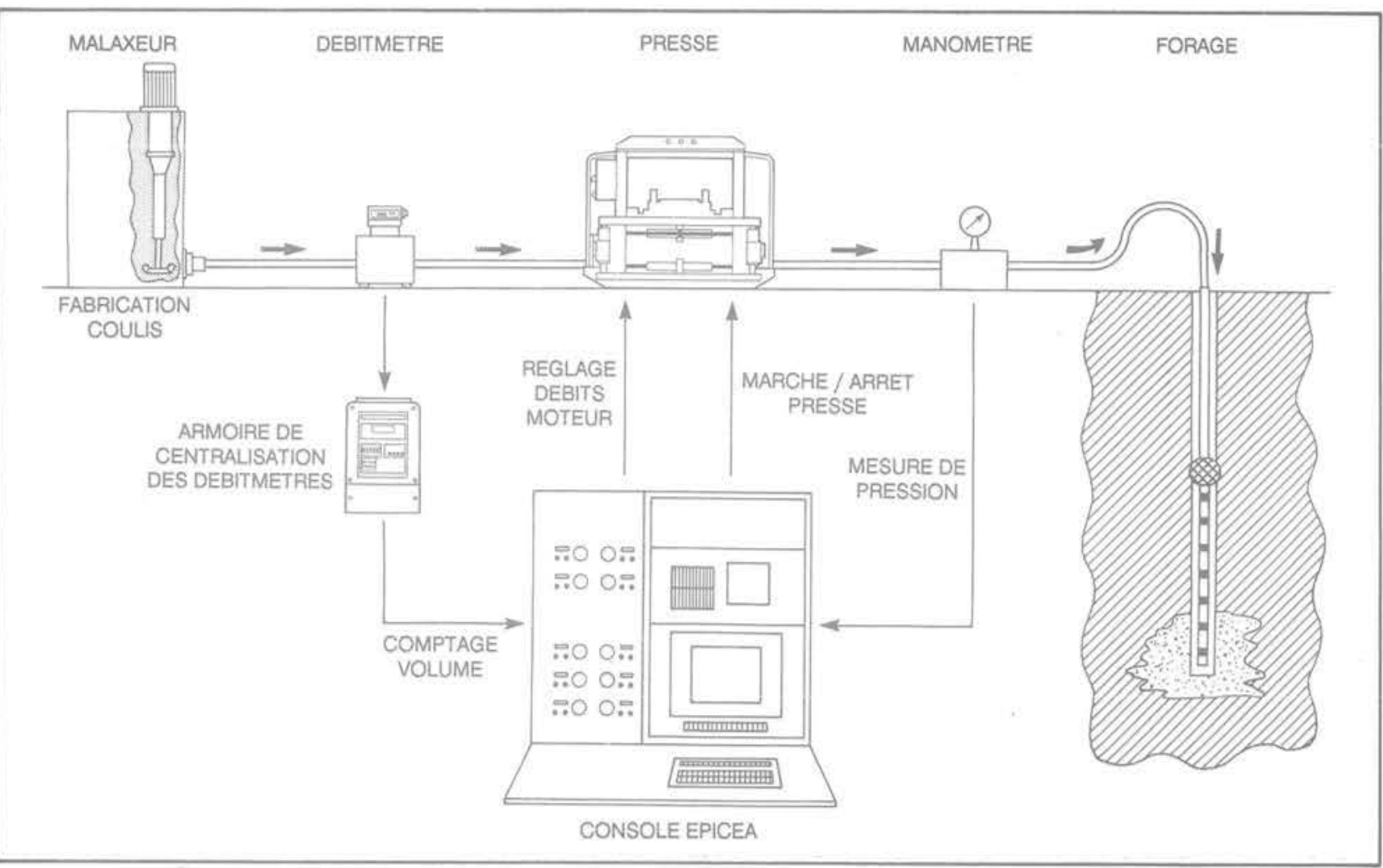

Fig. 11. - Système EPICEA chaîne de mesure.

Fig. 11, - EPICEA system acquisition chain.

et de respecter parfaitement des consignes d'injection aussi compliquées soient elles.

L'opérateur voit en permanence sur son écran et pour chaque point d'injection l'enregistrement des cinq minutes d'injection qui précèdent sous forme de courbes (pression, volume et débit), les valeurs instantanées, les critères d'injection ainsi que les coordonnées géométriques (forage, tranche et profondeur). En mode automatique, à chaque intervention de l'ordinateur, un message est affiché pour l'opérateur, numéro de pompe et raison de l'arrêt; l'opérateur peut ainsi se concentrer sur d'autres tâches d'évaluation de l'injection et anticiper les arrêts conduisant ainsi à une meilleure gestion des opérations d'injection.

Au niveau du traitement des données, la figure 12 décrit la chaîne d'information en cormmençant par la génération des consignes d'injection qui peut être:

- manuelle,

- définie par un système expert alimenté par des enregistrements numériques de paramètres de forage,

- obtenue par un programme de CAO tri-dimensionnel dans les cas où la géométrie du traitement est complexe (auréoles dans des plans non perpendiculaires à l'axe d'un tunnel)

A chaque fin de poste ou une fois par jour, les données sont transférées sous forme de disquette sur un autre ordinateur où la base de données est mise à jour par un technicien. Outre un rapport journalier précis et immédiat et les courbes de pression débit et volume en fonction du temps pour chaque tranche, le logiciel d'exploitation comprend une base de données permettant d'extraire n'importe quel sous-groupe moyennant l'utilisation de jusqu'à 99 critères de sélection. Ceci permet de faire des analyses statistiques sur une population

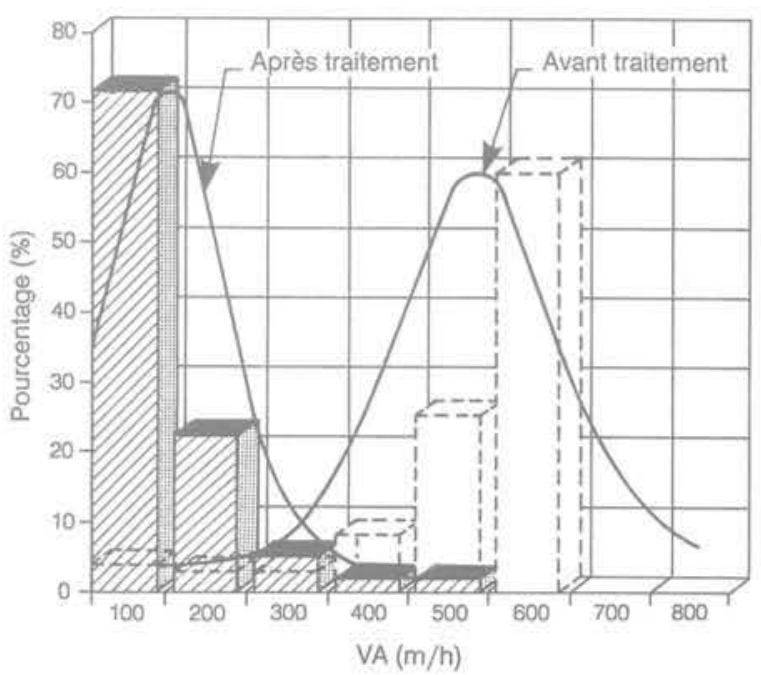

Fig. 13. - Evolution de la vitesse d'avancement de l'outil de forage au cours du traitement.

Fig. 13. - Evoluting of tool avvance velocity during treatment. 


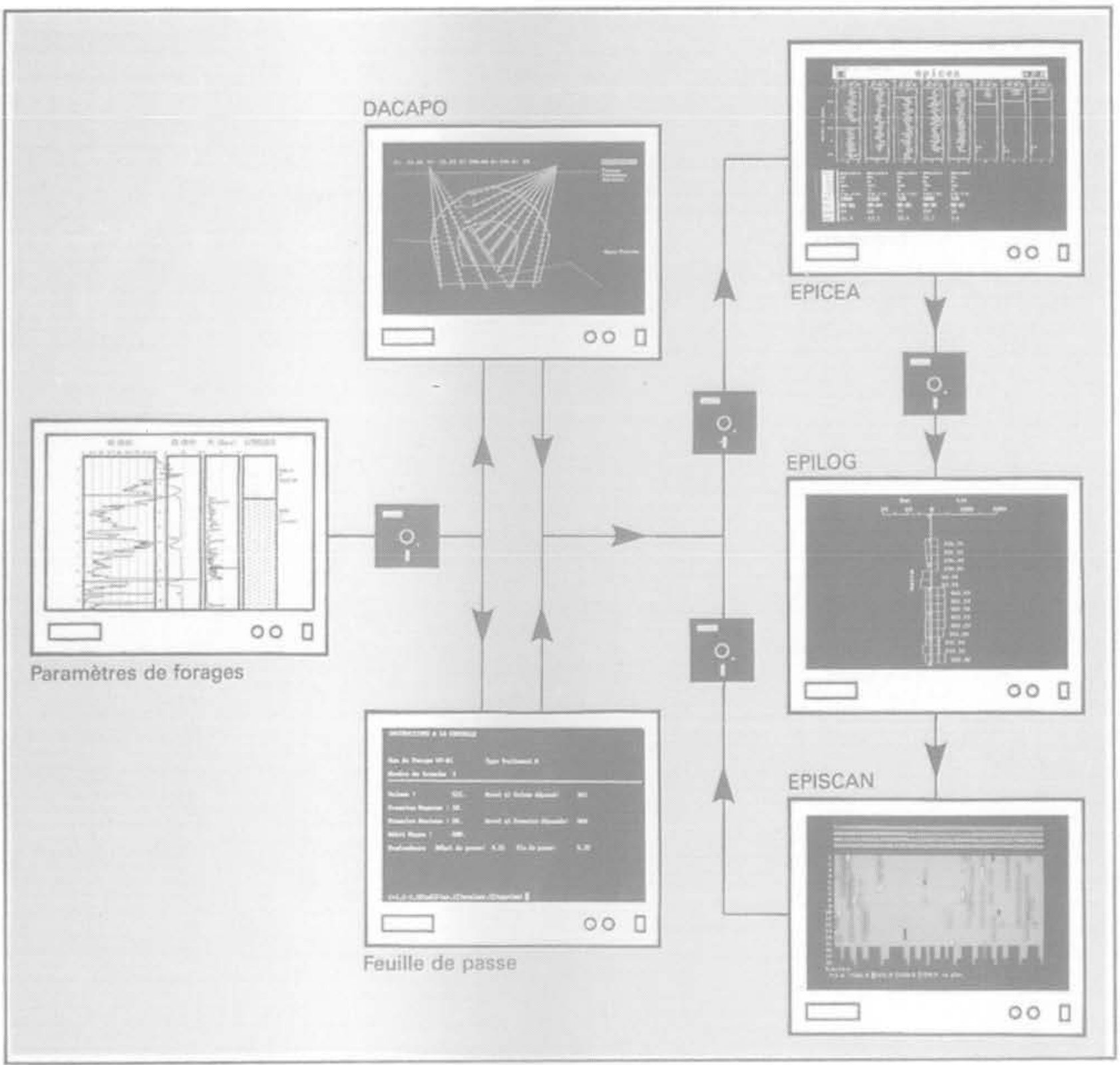

Fig. 12, - Système EPICEA chaîne de traitement informatique.

Fig. 12. EPICEA system data processing chain.

donnée tant au niveau des pressions (moyennes, maxima ou de fin d'injection) que des débits (moyens ou de fin d'injection) ou des volumes, et de dresser également des cartes de zones de comportement homogène qui sont autant d'aides à la décision dans la conduite du traitement. Ces aides à la décision, dans le cas précis qui nous intéresse, auraient été quasiment impossibles à obtenir manuellement au vu de la quantité d'informations à traiter: 500 à 600 tranches par jour avec simultanément au moins deux types de coulis.

D'autres moyens furent également mis en œuvre pour assurer la qualité du traitement et notamment le suivi de l'évolution des paramètres de forage. Plus particulièrement la vitesse instantanée d'avancement de loutil de forage, Va, qui est directement liée à poussée constante aux caractéristiques mécaniques du terrain a fait l'objet d'une analyse statistique. On a ainsi vu passer la Va de valeurs supérieures à $400 \mathrm{~m} / \mathrm{h}$ dans les zones décomprimées à $264 \mathrm{~m} / \mathrm{h}$ après le bourrage au mortier, $120 \mathrm{~m} / \mathrm{h}$ après linjection des primaires en bentoniteciment, $68 \mathrm{~m} / \mathrm{h}$ en fin d'injection, à comparer à $65 \mathrm{~m} / \mathrm{h}$ pour le terrain vierge. Un exemple typique de distributions de vitesses d'avancement au même endroit à des phases différentes est donné en figure 13. Ces résultats ont été confirmés par une série de 66 essais SPT réalisés au cours du traitement et qui ont vu les caractéristiques du terrain passer de $\mathrm{N}=5$ à $\mathrm{N}>50$ au coeur de la zone effondrée.

Enfin des échantillons des différents coulis ont été systématiquement prélevés et testés afin de vérifier la ré- 
sistance de ces derniers. Des essais de viscosité Marsh et de densité étaient également réalisés deux fois par jour pour chaque coulis.

\section{CONCLUSION}

A la fin du mois de septembre 1991, la réexcavation de la partie la plus critique en méthode allemande, à savoir les deux niveaux de galeries latérales et la demi-section supérieure (sans galerie de faîte) était terminée sans aucun problème.

Le système EPICEA associé à l'utilisation de coulis ap. propriés a permis de réaliser en 6 mois à l'aide de 14 presses des travaux qu'il n'était pas pensable de réaliser de manière traditionnelle au vu de l'exiguité de la plate-forme de travail, du matériel à y faire travailler et de la complexité du traitement 